\title{
PROJETO AEROGEOFÍSICO SERRA DO MAR SUL: UMA ABORDAGEM SEMI-QUANTITATIVA
}

\author{
DONAIDO C. SILVA*, ** \& MARTA S.M. MANTOVANI*
}

\begin{abstract}
SERRA DO MAR SUL AEROGEOPHYSICAL SURVEY: A SEMI-QUANTITATIVE ANALYSIS. Several airbome surveys have been promoted in Brazil by the Departamento Nacional da Produção Mineral (DNPM). Nevertheless, due to DNPM priorities, the obtained flown records are not optimized to allow more than semi-quantitative levei details. The methodology and data processing of airborne gamma-ray spectrometry in Brazil, hás not reached the evolution obtained in other countries, mainly due to brazilian peculiar geological characteristics, which often limit data interpretation. Here, a re-evaluation of Serra do Mar Sul airborne gamma-ray spectrometry data is presented. Flown profiles records were re-processed, optimized and tested for potentiality analysis. Correlations between countings in different recording channels were highly improved by the adopted procedures. A terrestrial survey, using a portable gamma-ray spectrometer calibrated at the Comissão Nacional de Energia Nuclear (CNEN- RJ), was performed in order to establish a correspondence between counts and concentration for $\mathrm{U}$, Th and $\mathrm{K}$
\end{abstract}

Keywords: Gamma-spectrometry, aerial survey, natural radioactivity.

RESUMO O Departamento Nacional da Produção Mineral (DNPM) promoveu vários levantamentos aéreos (DNPM 1984) recobrindo grande parte do território nacional; entretanto, em relação ao potencial de informacões disponíveis, pouco foi feito no sentido de otimizar a utilização deste potencial em termos semiquantitativos. No Brasil, a evolucão da metodologia de aquisição e tratamento de dados aerogama espectrométrícos tem sido pouco avaliada, para a diversidade de condicionantes ambientais e operacionais das diferentes regiões do território nacional. Apresenta-se aqui uma reavaliação e o reprocessamento dos dados do Levantamento Serra do Mar Sul visando seu potencial de aplicação semi-quantitativo. O procedimento adotado apresentou sensível melhora nas correlações entre os canais de contagem, eliminado tendências regionalizadas não-relacionadas com a geologia. Para fins de semi-quantização, foram executadas medidas gama-espectrométricas de superfície, utilizando equipamento portátil calibrado sobre blocos do Instituto de Radioproteção e Dosimetria (IRD), da Comissão Nacional de Energia Nuclear (CNEN), Rio de Janeiro.

Palavras-chave: Aerogamaespectrometria, levantamento aéreo, radioatividade natural.

INTRODUÇ̃̃O A espectrometria de raios gama aerotransportada tem sido utilizada no Brasil na localização de áreas potenciais para a prospecção mineral. Visando o reconhecimento aéreo do máximo de área no território nacional dentro dos recursos disponíveis, o DNPM promoveu, no período de 1952 a 1980 , vários levantamentos aéreos (DNPM 1984), dentre os quais o Projeto Serra do Mar Sul, objeto deste trabalho. Em vista das metas prioritárias do DNPM, além do tratamento convencional utilizado rotineiramente para a redução dos dados a mapas de contorno, pouco foi feito no sentido de otimizar a informação contida nos registros das linhas voadas, de forma a permitir uma análise mais precisa e, conseqüentemente, um possível detalhamento semi-quantitativo. $E$ intuito deste trabalho apresentar uma reavaliação dos dados do projeto em questão, os quais foram reprocessados visando sua otimização para adequá-los aos testes de avaliação da potencialidade de análise.

O projeto recobre a área de exposição de rochas précambrianas nos Estados do Paraná, de Santa Catarina, e parte do Estado de São Paulo (Fig. 1). Constitui a única fonte de dados radiométricos e magnetométricos recobrindo extensivamente a região. Foi executado pela GEOFOTO S.A., no período de 1973 a 1976, contratada pela Companhia de Pesquisa de Recursos Minerais (CPRM) (GEOFOTO 1978). As considerações que seguem visam prestar subsídio ao estudo da distribuição superficial do $\mathrm{K}, \mathrm{U}$ e Th, de suas aplicações na identificação de variações litológicas e na delimitação de áreas favoráveis à exploração mineral.
DESCRICÃO DO AEROLEVANTAMENTO E DA AQUISIÇÃO DOS DADOS GAMA-ESPECTROMÉTRICOS Os equipamentos e os procedimentos utilizados na fase de levantamento, bem como as correções aplicadas aos dados, são fatores condicionantes da qualidade e da aplicação dessa metodologia para os objetivos propostos. Em vista disso, reproduzimos resumidamente a descrição das características operacionais do levantamento (Tab. l, GEOFOTO 1978).

A correção dos dados digitais, realizada após a fase de aquisição, levou em consideração a radiação de fundo, o espalhamento Compton e a atenuação atmosférica, conforme GEOFOTO (1978), descrito a seguir:

(a) a determinação do nível da radiação cósmica, que constitui uma das componentes da radiação de fundo, foi baseada nos níveis dos quatro canais obtidos diariamente em voos teste na altura de $750 \mathrm{~m}$, realizados no início e ao término da cada voo. Após introdução dessa correção nos registros espectrométricos, foram verificados os valores dos quatro canais sobre rios e grandes açudes com o objetivo de avaliar se a radiação residual sobre esses pontos foi reduzida praticamente a zero.

(b) a correção do efeito devido ao espalhamento Compton utilizou as fórmulas seguintes

$$
\mathrm{U}_{\text {corr }}=\mathrm{U}-\alpha \mathrm{Th} \quad \mathrm{K}_{\text {corr }}=\mathrm{K}-\beta \mathrm{Th}-\mathrm{U}_{\text {corr }}
$$

onde $\mathrm{U}, \mathrm{K}$, e Th são os valores da radiação gama nesses canais, subtraídas as contribuições da radiação de fundo, tendorse utilizado como coeficientes de dispersão Compton:

\footnotetext{
* Instituto Astronómico e Geofísico, Universidade de São Paulo, Rua do Matão 1226, CEP 05508-900, São Paulo, SP, Brasil, e-mail: marta@iag.usp.br ** MINEROPAR, Rua Constantino Marochi, 800, CEP 80030-360, Curitiba, PR, Brasil
} 


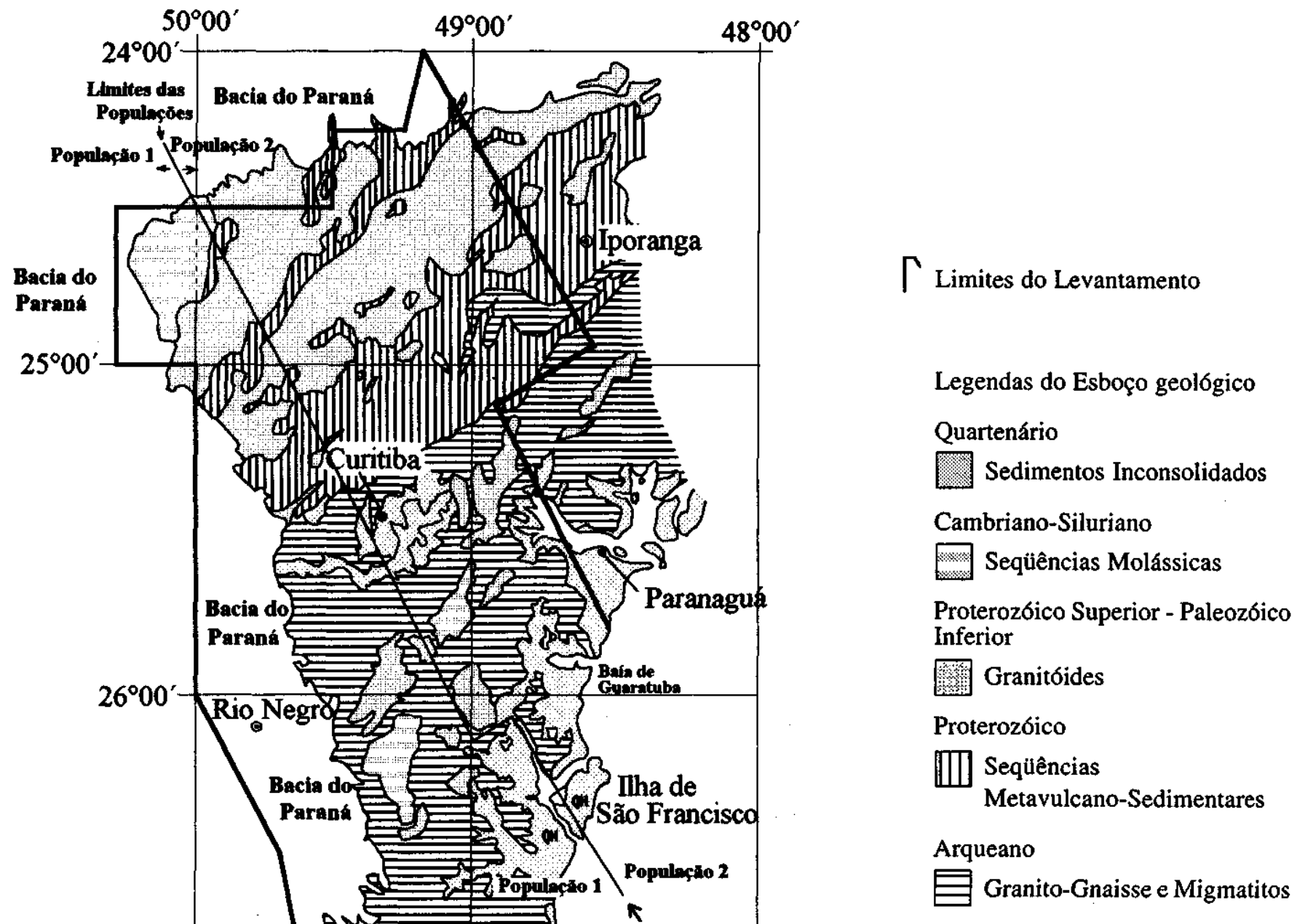

Esboço geológico simplificado da Carta Geológica do Brasil ao Milionésimo MME-DNPM 1974

Figura 1 - Esboço geológico da área de recobrimento do Projeto Aerogeofísico Serra do Mar Sul Figure 1 - Geological sketch map showing the área surveyed through the Projeto Aerogeofisico Serra do Mar Sul 
Tabela 1- Características operacionais e descrição dos equipamentos

Table 1 - Operational characteristics and instruments description

\begin{tabular}{|c|c|}
\hline Área total & $50.000 \mathrm{~km}^{2}$ \\
\hline Extensão total das & $53.924 \mathrm{~km}$ \\
\hline $\begin{array}{l}\text { Espaçamento entre } \\
\text { linhas de vôo }\end{array}$ & $\begin{array}{l}\text { nominal compreendido entre } 750 \\
\text { a } 1.250 \mathrm{~m} \text {, localmente ocorrem } \\
\text { sobreposições ou afastamentos } \\
\text { superiores a } 2 \mathrm{~km}\end{array}$ \\
\hline Altura de vôo & $\begin{array}{l}\text { nominal de } 150 \mathrm{~m} \text {; altura média } \\
\text { de } 158 \mathrm{~m} \text {, variando entre } 80 \text { a } \\
\text { mais de } 300 \mathrm{~m}\end{array}$ \\
\hline $\begin{array}{l}\text { Volume total dos } \\
\text { cristais }\end{array}$ & 16,681 \\
\hline Tempo de integraçāo & 1 segundo (c.a. $58 \mathrm{~m}$ ) \\
\hline $\begin{array}{l}\text { Direção das linhas } \\
\text { de vốo }\end{array}$ & N30W \\
\hline $\begin{array}{l}\text { Canais utilizados } \\
\text { tório e campo } \\
\text { magnético }\end{array}$ & contagem total, potássio, urânio, \\
\hline $\begin{array}{l}\text { Equipamento de } \\
\text { detecção }\end{array}$ & $\begin{array}{l}\text { Espectrômetro modelo DGRS- } \\
3001 \text {, da Exploranium, acoplado a } \\
\text { um conjunto de nove cristais de } \\
\text { NaI(T1), com formato cilíndrico } \\
\text { de 6" x 4", totalizando } 1017,88 \\
\text { polegadas cúbicas detecção }\end{array}$ \\
\hline $\begin{array}{l}\text { Equipamento de } \\
\text { registro radiométrico }\end{array}$ & $\begin{array}{l}\text { Rádio altímetro tipo STR, modelo } \\
54 \mathrm{~B} \text {, com precisão a } 122 \mathrm{~m} \text { de } \\
\text { altura da ordem de } 5 \%\end{array}$ \\
\hline Posicionamento & $\begin{array}{l}\text { Baseado em registros fotográficos } \\
\text { obtidos na frequîência de um } \\
\text { quadro cada dois segundos por } \\
\text { uma câmara Vinten II para filme } \\
\text { de } 35 \mathrm{~mm}\end{array}$ \\
\hline Navegaçäo & visual baseada em fotomosáicos \\
\hline
\end{tabular}

Tabela 2 - Intervalos de energia utilizados para os canais de contagem

Table 2 - Energy intervals for U, Th, K, and Total countings

\begin{tabular}{l|l}
\hline Canal de contagem & Limites das janelas \\
\hline Contagem total & $1,00-2,90 \mathrm{MeV}$ \\
\hline Potássio & $1,35-1,65 \mathrm{MeV}$ \\
\hline Urânio & $1,65-2,30 \mathrm{MeV}$ \\
\hline Tório & $2,30-2,90 \mathrm{MeV}$ \\
\hline
\end{tabular}

$$
\alpha=0,365 \quad \beta=0,50 \quad \gamma=0,77
$$

(c) a correção de altura foi feita convertendo-se as contagens em cada ponto amostrado para a altura nominal de voo $(\mathrm{N})$ com a utilização da fórmula

$$
\mathrm{N}_{150}=\mathrm{N} \mathrm{e} e^{\mu(\mathrm{h}-150)}
$$

onde $\mathrm{N}$ é a contagem obtida após as correções da radiação de fundo e do efeito Compton, $h$ é a altura do sensor em relação ao solo, e é o coeficiente de atenuação atmosférica. Os coeficientes de atenuação utilizados obtidos em Darnley $\&$ Grasty (1971) foram:

$\mu$ total $=6,6 \times 10 \mathrm{~m}$ $\mu$ urânio $=5,6 \times 10 \mathrm{~m}$

$$
\begin{aligned}
& \text { upotássio }=7,6 \times 10 \mathrm{~m} \\
& \mu \text { Utório }=5,6 \times 10 \mathrm{~m}
\end{aligned}
$$

\footnotetext{
CONSIDERAÇÕES SOBRE OS DADOS UTILIZA-

DOS São várias as fontes de erro que interferem nas
}

medidas radiométricas. Às variáveis normais relacionadas aos contrastes das propriedade físicas e da geometria, somam-se o tamanho e eficiência do detetor, a velocidade de deslocamento da aeronave, os efeitos das variáveis meteorológicas, as condicões de equilíbrio das séries de decaimento do urânio e do tório, e o comportamento químico do tório, urânio e do potássio no ambiente.

As características regionalizadas para as condições de amostragem, condicionadas pela grande extensão e geomorfologia da área, envolvem fatores tais como as variações de pressão, temperatura e umidade do ambiente, que interferem nos valores de contagens (espalhamento Compton, atenuação da radiacão na atmosfera e no solo) e as regiōes acidentadas que implicam em grandes variações na altura de voo. Como fator adicional, tem-se a utilização de duas bases operacionais - o Aeroporto de Florianópolis e Aeroporto do Bacacheri, em Curitiba - envolvendo diferentes condições atmosféricas e de radiação de fundo nos testes de calibraçã̃o.

Outros aspectos a serem levados em consideração são o espaçamento entre as linhas de voo, variando em geral entre $750 \mathrm{e} 1.250 \mathrm{~m}$; porém, localmente ocorrem sobreposiç̃es ou afastamentos superiores a dois quilómetros, e as coordenadas UTM dos registros cujos valores são incompatíveis com o posicionamento geográfico dos perfis, fato observável nos mapas de linhas de voo. Esta distorção geo-referencial varia regionalmente, da ordem de vários metros próximo ao limite oeste do levantamento, na latitude de Curitiba, aumentando de forma centrífuga, até valores superiores a quatro quilómetros. Areas com relevo muito acidentado, nas quais a altura de voo é muito variável, às vezes superiores a $300 \mathrm{~m}$, constituem fatores a serem levados em consideração na utilização dos dados.

Quanto a radiação de fundo, são três as fontes que interferem nos dados espectrométricos aerotransportados: a radioatividade proveniente da aeronave, a radiação cósmica, e o radônio atmosférico. Destas, o radônio causa as maiores interferências, sendo liberado do solo por processos de difusão, cujas taxas variam em função da pressão atmosférica, da umidade do solo, das características do material que recobre o terreno, dos ventos e da temperatura ambiental. Seus produtos de decaimento, $\mathrm{Pb}^{214}$ e $\mathrm{Bi}^{214}$, são fixados aos aerossóis e suas distribuições dependem do movimento das massas de ar na atmosfera. Grasty (1979) observou que, entre localidades distantes poucos quilómetros entre si, no início do dia, com o aumento da movimentação das massas de ar atmosférico, estas concentrações tendem à dispersão. A grande extensão e diversidade físiográfica da área levantada resultaram em interferências regionalizadas nas taxas de contagens, contribuindo em grau variável para a composição da radiação de fundo, dependendo da distribuição do radônio atmosférico ao longo do período de voo.

Considere-se também a aleatoridade do processo de desintegração radioativa, cujo desvio padrão é associado à medida igual a raiz quadrada da contagem obtida, e, ao se aplicarem as correções para a subtração da radiação de fundo e do efeito Compton, que envolvem uma medida com os respectivos erros associados, o erro resultante é incrementado (Killeen 1979). Na subtração da radiação de fundo, por exemplo, 0 erro na contagem corrigida será:

$$
\pm \sigma_{\mathrm{c}}=\left(\sigma_{\mathrm{b}+\sigma_{\mathrm{bg}}}^{2}\right)^{1 / 2}
$$

Onde $\mathrm{a}_{\mathrm{c}}$ é o desvio padrão da contagem após a subtração da radiação de fundo, $a_{b}$ o desvio padrão da contagem bruta e $a_{b p}$ o desvio padrão da contagem da radiação de fundo. Sendo que, para as condiç̃es de amostragem, o volume dos cristais utilizado é considerado pequeno e, dependendo dos teores de radioelementos das litologias sobrevoadas, às taxas de contagens muito baixas estão associados maiores erros. Como referencial, a tabela 3 relaciona os valores médios dos dados de contagem do projeto e a ordem das incertezas envolvidas. 
Estas implicam em limitações no tratamento e interpretação dos dados, notadamente para amostras com baixos teores de $\mathrm{Th}, \mathrm{U}$ e K; rochas ou processos relativamente enriquecidos nestes elementos são definidas com maior segurança.

Tabela 3 - Estimativas dos erros para os valores médios de contagens do projeto

Table 3 - Average counting error estimative

\begin{tabular}{l|c|c|c|c}
\hline \multirow{2}{*}{$\begin{array}{l}\text { Canal de } \\
\text { contagem }\end{array}$} & \multicolumn{2}{|c|}{$\begin{array}{c}\text { Contagens sem } \\
\text { correçöes }\end{array}$} & \multicolumn{2}{c}{ Contagens corrigidas } \\
\cline { 2 - 5 } & $\begin{array}{c}\text { Valor de } \\
\text { contagem }\end{array}$ & $\begin{array}{c}\text { Desvio } \\
\text { Padrão }\end{array}$ & $\begin{array}{c}\text { Valor de } \\
\text { contagem }\end{array}$ & $\begin{array}{c}\text { Desvio } \\
\text { Padrăo }\end{array}$ \\
\hline Total & $519 \pm 23 \mathrm{cps}$ & $4,4 \%$ & $226 \pm 13 \mathrm{cps}$ & $12,6 \%$ \\
\hline Potássio & $103 \pm 10 \mathrm{cps}$ & $9,8 \%$ & $29 \pm 12 \mathrm{cps}$ & $41,4 \%$ \\
\hline Urânio & $47 \pm 7 \mathrm{cps}$ & $14,3 \%$ & $27 \pm 8 \mathrm{cps}$ & $29,6 \%$ \\
\hline Tório & $31 \pm 6 \mathrm{cps}$ & $18,1 \%$ & $24 \pm 6 \mathrm{cps}$ & $25,1 \%$ \\
\hline $\begin{array}{l}\text { Altura média } \\
158 \text { de vốo }\end{array}$ & \multicolumn{4}{|c}{158} \\
\hline $\begin{array}{l}\text { Número total } \\
\text { de perfis }\end{array}$ & \multicolumn{4}{|c}{552} \\
\hline
\end{tabular}

ANÁLISE E CONSISTÊNCIA DOS DADOS O grande número de fatores envolvidos no projeto pelo seu porte, a disponibilidade de informações pertinentes à fase de levantamento e a quantidade de informações levantadas conduziram para uma abordagem analítica, baseada em valores médios das linhas de voo.

Nos levantamentos aéreos, as médias de contagens das linhas de voo geralmente apresentam altos coeficientes de correlação linear entre os canais. Tais resultados são esperados quando o levantamento envolve amplas regiões, tendendo a média para composição equivalentes a média crustal, sendo suavizadas as razões das variações locais. No Projeto Serra do Mar Sul, os coeficientes obtidos para a correlação linear entre canais de contagens são baixos, notadamente as relações potássio-urânio $(0,21)$ e potássiotório $(0,37)$. Nas figuras 2,3 e 4 , as relações entre os valores

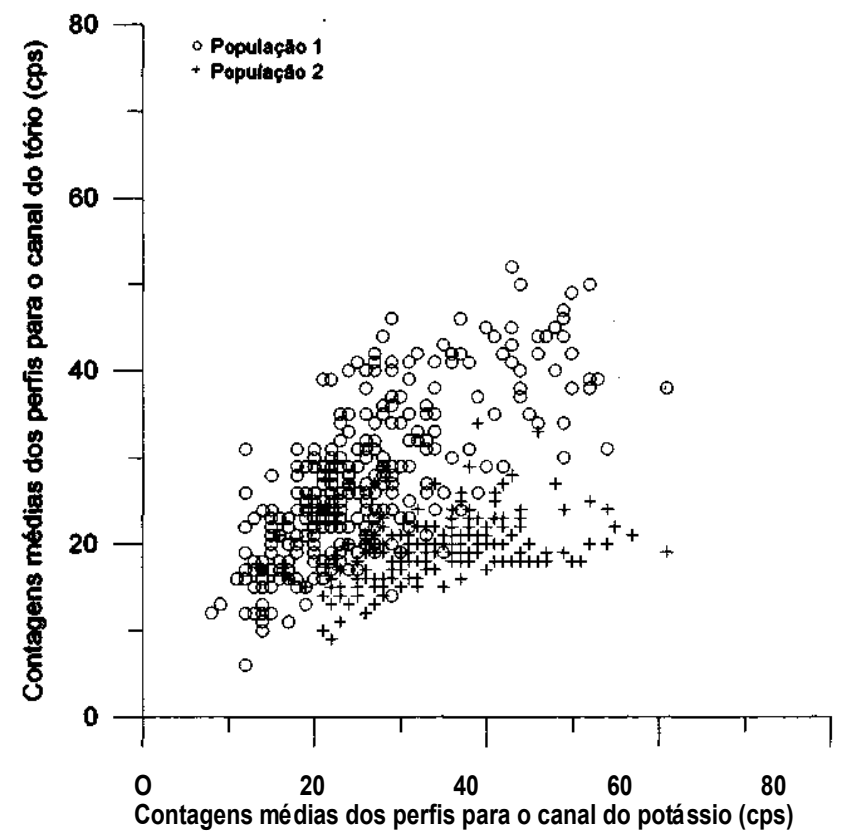

Figura 2 - Correlação entre os dados médios das linhas de voo para os canais do potássio e do tório. Dados originais para as duas populações

Figure 2 - Potassium and thorium mean data correlation on flight lines, for original data

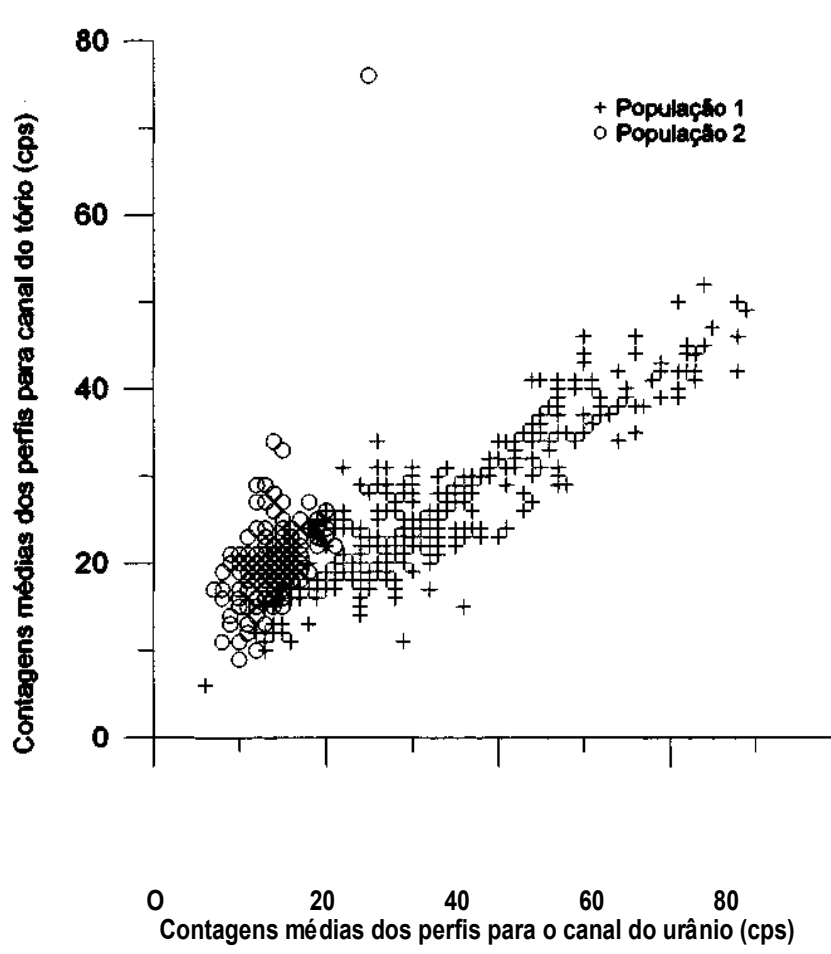

Figura 3 - Correlação entre os dados médios das linhas de voo para os canais do urânio e do tório. Dados originais para as duas populações

Figure 3 - Uranium and thorium mean data correlation on flight lines, for original data

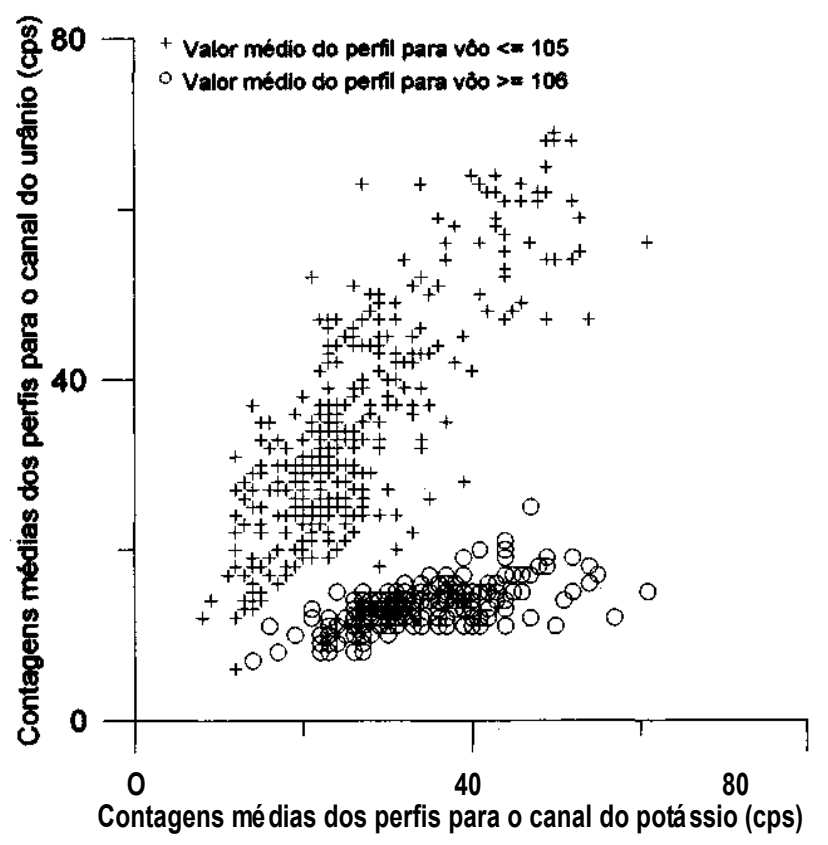

Figura 4-Correlação entre os dados médios das linhas de voo para os canais do potássio e do urânio. Dados originais para as duas populações

Figure 4 - Potassium and uranium mean data correlation on flight lines, for original data

médios dos perfis para esses canais sugerem duas tendências. O valor médio do canal do tório para a linha 222 , vôo 127 , de $76 \mathrm{cps}$, constitui o ponto mais isolado nos gráficos, $\mathrm{o}$ qual está relacionado com as duas maiores anomalias radio- 
métricas do sul do pais: os corpos carbonatíticos da Barra do Itapirapuã e de Mato Preto, ambos da fase tardia de diferenciação, com excepcional enriquecimento em tório. Esta linha de voo foi excluída dos cálculos.

No conjunto dos dados médios, as relações entre canais sugerem duas "populações" (Tab. 4). Razões potássio-urânio inferiores à unidade $(\mathrm{K} / \mathrm{U}<1)$, potássio-tório próximas a esta $(\mathrm{K} / \mathrm{Th} \sim 1)$ e urânio-tório superiores $(\mathrm{U} / \mathrm{Th}>1)$ são comuns aos perfis compreendidos pêlos voos de número inferior a 106. Voos iguais ou superiores ao 106 têm comportamento inverso, ou seja, as razões potássio-urânio e potássio-tório são superiores à unidade e as razões urâniotório são inferiores. Tendo em vista o resultado, a totalidade dos dados foi subdividida em duas "populações", sendo a população 1 para os voos com número inferior ao 106, e a população 2 para os demais voos (Tab. 5).

Tabela 4 - Médias dos canais de contagens (corrigidas) para as populações

Table 4 - Counting average value corrected for each population

\begin{tabular}{c|c|c|c|c|c}
\hline População & $\begin{array}{c}\text { Contagem } \\
\text { Total }\end{array}$ & $\begin{array}{c}\text { Canal } \\
\text { do K }\end{array}$ & $\begin{array}{c}\text { Canal } \\
\text { do U }\end{array}$ & $\begin{array}{c}\text { Canal } \\
\text { do Th }\end{array}$ & $\begin{array}{c}\text { Altura } \\
\text { de vố }\end{array}$ \\
\hline 1 & 212 & 22 & 33 & 26 & 149 \\
\hline 2 & 226 & 33 & 13 & 20 & 177 \\
\hline
\end{tabular}

Tabela 5-Razões entre canais de contagens para as populações

Table 5 - Channels counting ratios for the defined populations

\begin{tabular}{l|l|l}
\hline $\begin{array}{l}\text { Razōes entre canais para } \\
\text { os dados corrigidos }\end{array}$ & \multicolumn{2}{|c}{ População } \\
\cline { 2 - 3 } & 1 & 2 \\
\hline CT/K & 9,64 & 6,85 \\
\hline CT/U & 6,42 & 17,38 \\
\hline CT/Th & 8,15 & 11,30 \\
\hline $\mathrm{K} / \mathrm{U}$ & 0,66 & 2,54 \\
\hline $\mathrm{K} / \mathrm{Th}$ & 0,85 & 1,65 \\
\hline $\mathrm{U} / \mathrm{Th}$ & 1,27 & 0,65 \\
\hline
\end{tabular}

No tratamento individual de cada conjunto de dados, os coeficientes de correlação da população 1 se apresentam consideravelmente melhores (Tab. 6) e próximos ao esperado para esse tipo de levantamento. A população 2 apresenta, persistentemente, baixos coeficientes.

Tabela 6 - Coeficientes de correlação linear entre canais, utilizando dados médios das linhas de vôo

Table 6 - Linear correlation coefficients between channels for flown lines mean values

\begin{tabular}{l|l|l|l|l|l|l}
\hline Populaçāo & \multicolumn{5}{|c}{ Coeficientes de correlaçāo linear (contagens corrigidas) } \\
\hline & CT x K & CT x U & CT x Th & K x U & K x Th & U x Th \\
\hline 1 e 2 & 0,76 & 0,65 & 0,82 & 0,21 & 0,37 & 0,83 \\
\hline 1 & 0,85 & 0,97 & 0,93 & 0,81 & 0,74 & 0,91 \\
\hline 2 & 0,78 & 0,76 & 0,79 & 0,62 & 0,37 & 0,51 \\
\hline
\end{tabular}

Uma vez definidos os limites de cada população, foram selecionadas 47 linhas de voo, sendo 21 pertencentes à população 1 e 26 à população 2 , prosseguindo-se na reavaliação dos dados. As duas populações foram delimitadas para uma região relativamente homogénea em suas características geológicas e geomorfológicas, situada no Primeiro
Planalto Paranaense, a noroeste de Curitiba. Os resultados obtidos para os valores médios das linhas nesta região, relacionados nas tabelas 7 e 8 , confirmam os resultados obtidos.

Tabela 7-Médias dos canais de contagens corrigidas para as populações limitadas a NW de Curitiba

Table 7 - Counting channels average corrected for population at NW of Curitiba

\begin{tabular}{l|l|l|l|l|l}
\hline População & CT & K & U & Th & Altura de vôo \\
\hline 1 & 189 & 14 & 29 & 26 & 140 \\
\hline 2 & 257 & 40 & 15 & 21 & 156 \\
\hline
\end{tabular}

Tabela 8 - Razões entre os canais de contagens corrigidas para as populações delimitadas à $N W$ de Curitiba Table 8 - Corrected counting ratios for population at NW of Curitiba

\begin{tabular}{l|l|l|l|l|l|l} 
População & CT/K & CT/U & CT/Th & K/U & K/Th & U/Th \\
\hline 1 & 13,50 & 6,52 & 7,27 & 0,48 & 0,54 & 1,11 \\
\hline 2 & 6,42 & 17,13 & 12,29 & 2,66 & 1,90 & 0,71 \\
\hline
\end{tabular}

As possíveis causas que determinaram a compartimentação dos dados do levantamento podem estar relacionados a um ou mais fatores. Na fase de levantamento, foram utilizadas duas bases de operações com elevações muito distintas em relação ao nível do mar, implicando em diferenças nas condições de obtenção da radiação de fundo (pressão atmosféríca, umidade relativa do ar, contribuição da radiação cósmica), sendo as diferenças de altitude da ordem de $800 \mathrm{~m}$. Não foi possível recuperar informações relacionando os voos às suas respectivas bases; no entanto, as relações entre os valores da radiação de fundo calculados para o canal de contagem total mostram certa compartimentação, bem como as do potássio e do urânio apresentam variações, embora menos acentuadas no limite entre as populações. A distribuição geográfica dos perfis sugere, para a população 1, a base no Aeroporto de Florianópolis, e, para os voos com números superiores, a base em Curitiba.

Considera-se que, por motivos operacionais condicionados ao posicionamento geográfico das bases, os procedimentos de calibração desenvolveram-se sob condições distintas. Os testes diários em terra estariam sujeitos às variações locais da radiação de fundo características para cada base. Essas variações podem ser relacionadas aos teores de tório, urânio e potássio dos materiais que constituem o piso e a região circundante dos locais de calibração e com a contribuição cósmica distinta para cada base, sob diferentes condições atmosféricas e composições radiogeoquímicas.

REPROCESSAMENTO DOS DADOS E AVALIAČ̃̃ DOS RESULTADOS Objetivando o tratamento singular do projeto, foi utilizada a normalização dos dados para compensar as tendências regionalizadas. Devido às distintas condições de levantamento para cada população, em virtude das condições de calibração e dos parâmetros utilizados na correção, o conjunto de informações foi considerado como constituindo dois levantamentos distintos (populações $1 \mathrm{e}$ 2). Considerando essa premissa, os procedimentos foram baseados nas sensibilidades para os canais de contagens do tório, do urânio e do potássio determinados experimentalmente, dentro das limitações existentes, para cada população (Cordeiro da Silva 1994).

Entre os vários fatores que interferiram na determinação dos coeficientes de sensibilidade, estão a falta de precisão para se delimitar no terreno as faixas de recobrimento do 
sensor, devido a indisponibilidade dos filmes de rasteio dos vôos; a pouca exatidão dos pontos fiduciais das linhas de vôo em termos geo-referenciados; e a variação na altura de vôo. Essas últimas, entretanto, aparentam inconsistência por causa da grande variabilidade nos desníveis em relação às pequenas distâncias entre fiduciais (da ordem de $60 \mathrm{~m}$ ) sobre relevo relativamente pouco acidentado, sugerindo imprecisão dos dados altimétricos. Apesar da pouca precisão na localização da faixa de amostragem do sensor aerotransportado, a área permite estimativa das sensibilidades relativas, uma vez que as informações aéreas onde as fiduciais se interceptam são representativas de porções equivalentes do terreno (Fig. 5).

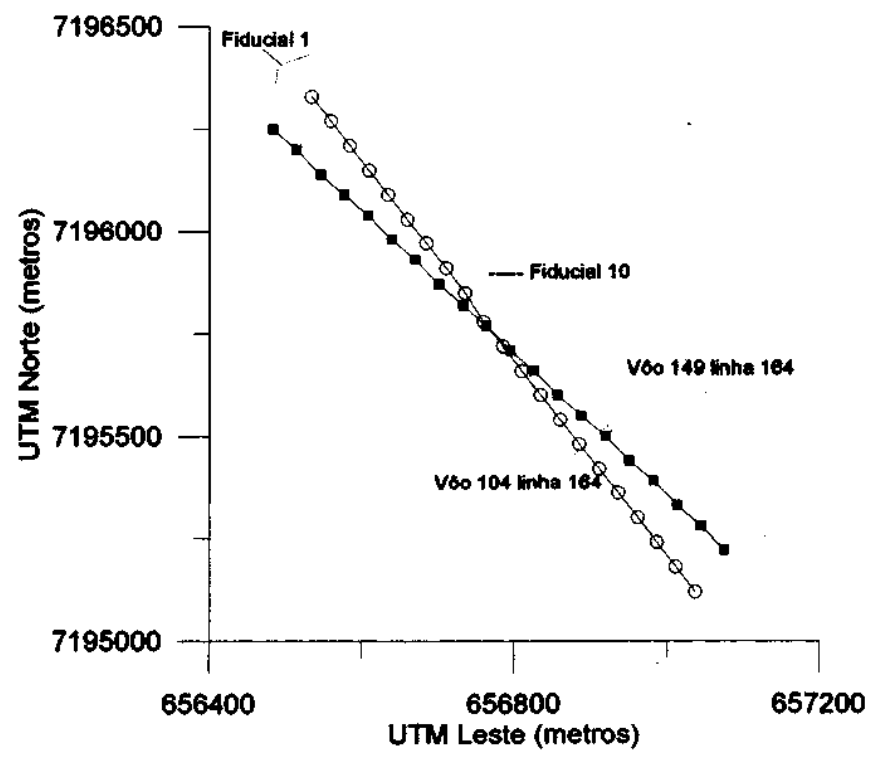

Figura 5 - Pontos fiduciais dos perfis utilizados na normalização dos dados na área de Campo Magro (PR) Figure 5 - Distribution of fiducial points for data normalization. Campo Magro, PR

Na obtenção dos coeficientes de sensibilidade, foram adotados os seguintes procedimentos:

(a) seleção de área padrão situada próxima a localidade de Campo Magro, no Município de Campo Largo (Paraná), onde o recobrimento florestal é incipiente e a litologia é relativamente homogénea (filitos), as faixas de amostragem da linha 164 voo 104 (população 1) e linha 164 voo 149 (população 2) se sobrepõem, e há relativa facilidade para a materialização aproximada das faixas de investigação do sensor aerotransportado no campo por causa das características fisiográficas da área; também condições adequadas para a determinação da radiação de fundo sobre corpo aquoso, para as medidas terrestres;

(b) calibração do gamaespectrômetro portátil para determinar os teores ao nível do solo: a calibração e determinação das sensibilidades do espectrômetro de raios gama portátil (GAD6 com sensor GSP3, do IAG-USP), baseadas em dados de contagens, foi obtida sobre blocos de calibração no Instituto de Radioproteção e Dosimetria (IRD), da Comissão Nacional de Energia Nuclear (CNEN), Rio de Janeiro blocos estes descritos em Barreto et ai (1986), efetuando-se medições durante 1.000 segundos, com o detector posicionado no centro e na superficie dos blocos de calibração; no cálculo dos coeficientes, foi aplicada metodologia transcrita em Grasty \& Darnley (1971);

(c) estimativas dos teores de tório, urânio e potássio na área padrão por espectrometria de raios gama utilizando o instru- mento portátil, com o sensor afastado $0,45 \mathrm{~m}$ do solo, sendo 24 pontos estacionários e nove perfis obtidos por caminhamentos, ambas com tempo de integração de 300 segundos; na amostragem, procurou-se evitar interferências antrópicas ocorridas no período entre os levantamentos aéreos (1976) e terrestre (1993), tendo como referência fotografias aéreas na escala 1:25.000 (de 1980), e possíveis contaminações por adubos químicos (potássio, e o urânio nos fertilizantes fosfatados) em áreas atuais de cultura vegetal; as porções cultivadas, constituindo diversas frações irregulares com dimensões inferiores a 1 hectare, determinaram distribuição irregular para os pontos amostrados, (d) determinação das relações contagem/teor para cada canal e respectivas populações, sendo os teores equivalentes expressos em partes por milhão de tório (eTh ppm) e urânio (eU ppm) e percentual de potássio $(\mathrm{K} \%)$. Em vista da irregularidade na distribuição dos dados terrestres e a falta de precisão na materialização da faixa de investigação no terreno (projeção das linhas de voo e dos pontos fiduciais no terreno), foram utilizados valores médios de contagens dos canais para os segmentos das linhas representativos da área amostrada e respectivos teores ao nível do solo. $\mathrm{O}$ intervalo utilizado nas estimativas, limitado pelas fiduciais 5 e 13 (Fig. 5), foi selecionado em função da superposição das linhas e da correlação das contagens entre os perfis. Estabelecidos os teores para a área padrão, foram determinados os coeficientes de sensibilidade (Tab. 9), isto é, a relação teor/contagem, para cada canal e respectiva população. Os teores equivalentes obtidos a partir destes coeficientes, estão diretamente relacionados às respostas espectrométricas em função das concentrações de radioelementos no terreno e independem das tendências inerentes às populações.

Esse procedimento resultou em coeficientes de correlação linear significativamente mais altos (Tab. 10).

Tabela 9- Sensibilidades estimadas para o espectrômetro aerotransportado e respectivas populações (para l cps) Table 9 - Estimated flown instrument sensibility for the two populations

\begin{tabular}{l|l|l}
\hline Elemento & População 1 & População 2 \\
\hline $\mathrm{K} \%$ & 0,012909 & 0,014388 \\
\hline $\mathrm{U}$ ppm & 0,027912 & 0,104613 \\
\hline Th ppm & 0,201036 & 0,384254 \\
\hline
\end{tabular}

Tabela 10-Relação entre os coeficientes de correlação e os dados médios das Unhas de vôo originais e após a normalização. Totalidade dos perfis do projeto

Table 10 - Correlation coefficients for both original and normalized average values; ali flown profiles are included

\begin{tabular}{l|l|c}
\hline Canais de correlaçāo & Dados originais & Dados normalizados \\
\hline $\mathrm{K} \times \mathrm{U}$ & 0,21 & 0,81 \\
\hline $\mathrm{K} \times \mathrm{T}$ & 0,37 & 0,73 \\
\hline $\mathrm{U} \times \mathrm{Th}$ & 0,83 & 0,85 \\
\hline
\end{tabular}

A distribuição dos pontos de amostragem no terreno e a pouca precisão na projeção dos pontos fiduciais não possibilitaram uma estimativa muito precisa para a sensibilidade dos equipamentos aerotransportados, resultando em teores aparentes, diante das deficientes condições de controle. Os parâmetros, obtidos a partir dos dados corrigidos de Campo Magro, resultaram no nivelamento para os canais do Th, U e K (médias dos perfis do projeto; Fig. 6, 7 e 8). 


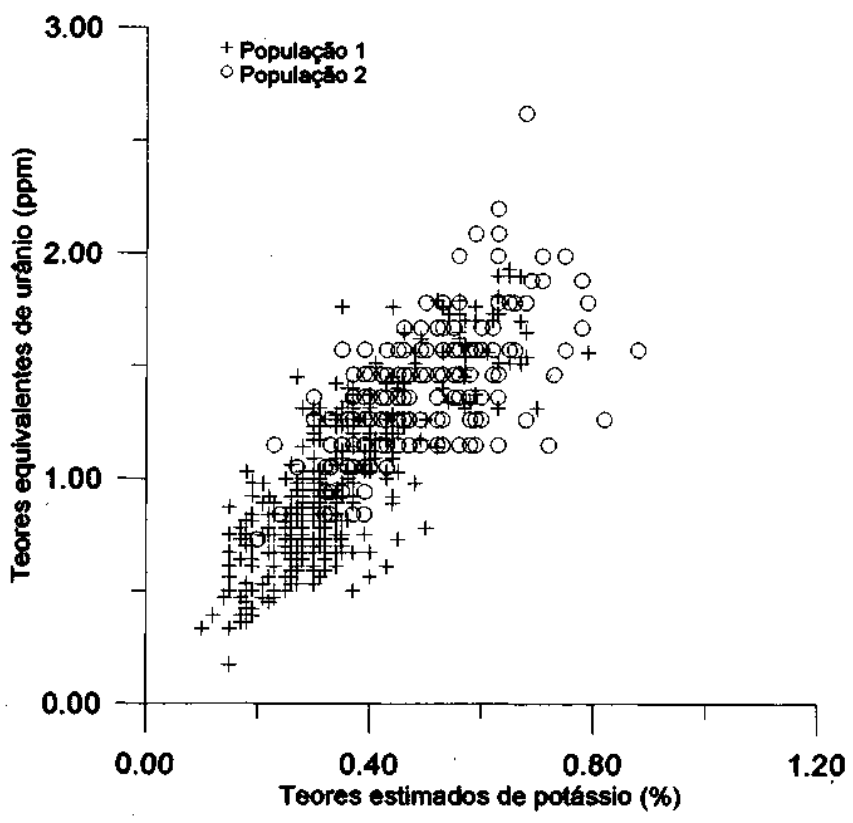

Figura 6 -Relação entre os dados médios das linhas de vôo normalizados em teores de potássio e urânio

Figure 6 - Relationship between fligth lines for potassium and uranium

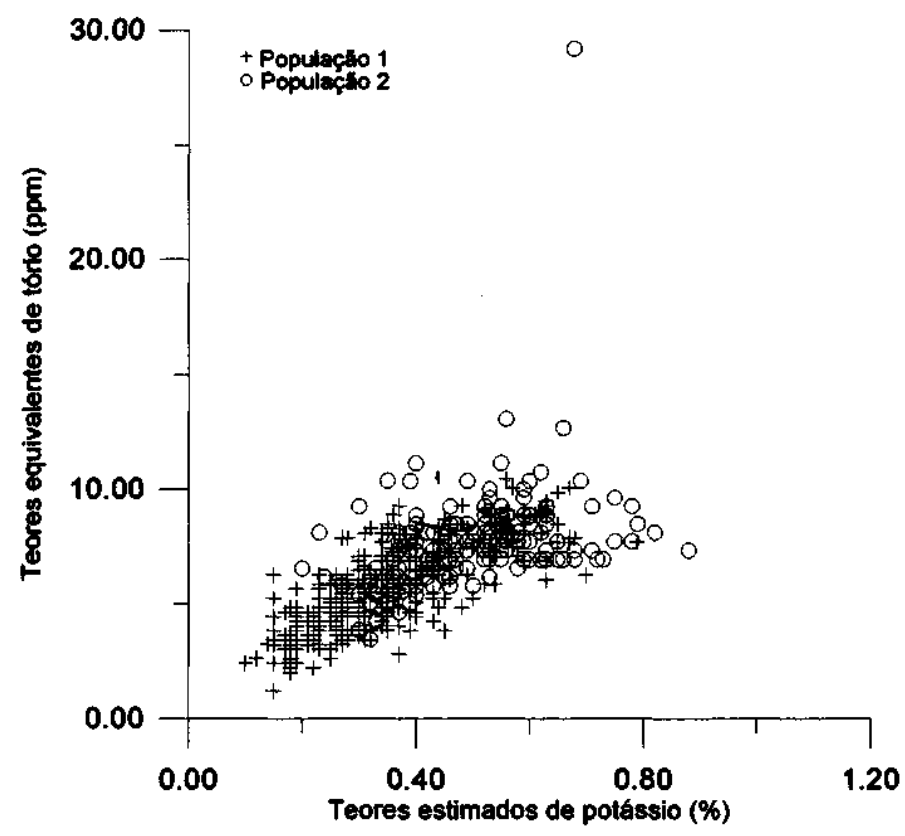

normalized mean values

Figura 7-Relação entre os dados médios das linhas de vôo normalizados em teores de potássio e tório

Figure 7 - Relationship between fligth lines for potassium and thorium normalized mean values

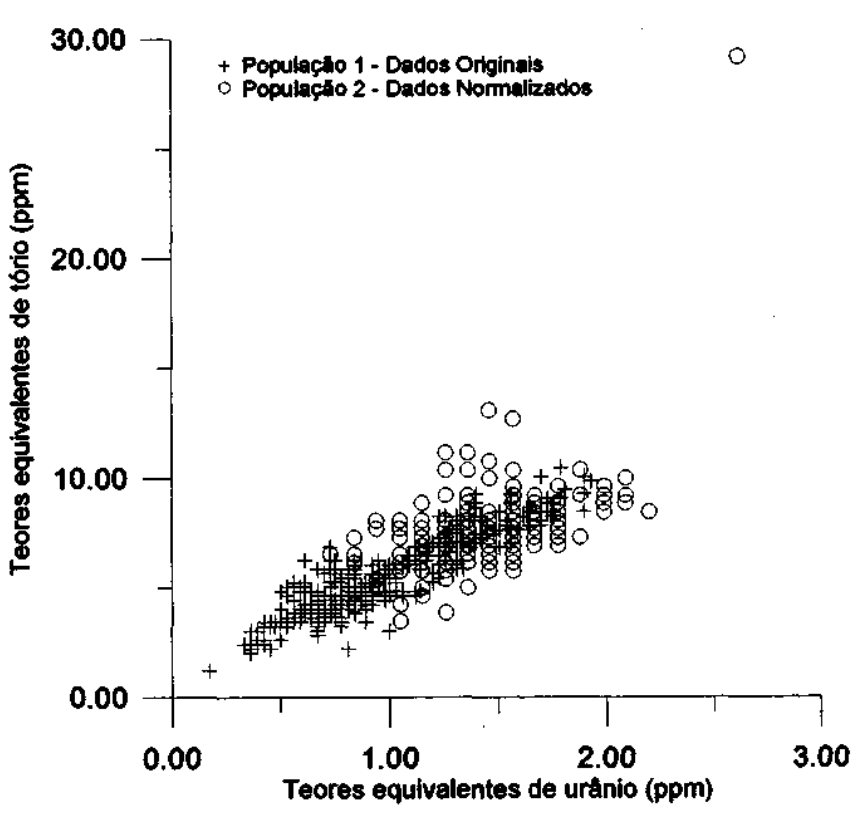

Figura 8-Relação entre os dados médios das linhas de voo normalizados em teores de urânio e tório

Figure 8 - Relationship between fligth lines for uranium and thorium normalized mean values

CONCLUSÕES Em termos de aplicação, os resultados obtidos são amplamente satisfatórios e, embora não tenham sido obtidos em condições ideais, o procedimento utilizado resultou numa sensível melhora das correlações entre os canais de contagens. Erros na normalização estão condicionados pelas diferentes alturas de voo entre os perfis utilizados, pela acurácia na determinação da radiação de fundo e de sua representatividade no período de amostragem (distinta para cada perfil), envolvendo ainda o grau efetivo de superposição entre a faixa de investigação e a área-padrão. A precisão nas determinações de campo, mesmo que a porção levantada não seja coincidente com a área de recobrimento mútuo dos perfis, pouco interfere na determinação dos fatores de normalização, em termos relativos entre as populações: esses fatores dependem principalmente do grau de superposição dos perfis, dos parâmetros de amostragem e redução dos dados originais, que por sua vez são dependentes dos coeficientes utilizados na correção da atenuação atmosférica, bem como dos erros estatísticos da contagens.

Agradecimentos Aos colegas F. Hiodo, W. Shukowsky, I. Braggion Jr., F. B. Ribeiro, E. Arioli, F. J. F. Ferreira e F. V. da Silva, pelo incentivo e sugestões recebidas, e a MINEROPAR, pelo apoio nos trabalhos de campo. O trabalho foi realizado com o apoio financeiro do $\mathrm{CNPq}$ e da FAPESP.

\section{REFERÊNCIAS BIBLIOGRÁFICAS}

BARRETO, P.M.C.; AUSTERLITZ, C; MALHEIROS, T; LOVBORG, L. 1986. Radioactive Concret Sources at IRD/CNEN, Brazilfor the Calibration of Uranium Exploration and Environmental Field Instruments. IRD/DEX-3/CNEN.

CORDEIRO DA SILVA, D. 1994. Espectrometria de Raios Gama Aplicada na Classificação de Granitóides. São Paulo. 83 p. (Dissertação de Mestrado, IAG-USP).
DARNLEY, A.G. \& GRASTY, R.L. 1971. Mapping from the air by gamma-ray spectrometry. Proceed., 3rd Int. Symp., Toronto, April 16-18, 1970, Geochemical Exploration. The Canadian Inst. of Mining and Metallurgy. Special vol. 11, pg. 485-500.

DEPARTAMENTO NACIONAL DA PRODUÇÃO MINERAL 1984. Inventário dos Levantamentos Aerogeofisicos Executados pelo $D N P M$. Brasília, DNPM. (Série Geologia 25, Seç̧ão Geofísica 3). 
GEOFOTO 1978. Projeto Serra do Mar Sul - Relatório Final GEOFOTO

Ltda. 120 p. Data Processing Techniques. Austrália, Bureau of Mineral

GRASTY, R.L. 1976. A calibration procedure for an airborne gamma-ray spectrometer. Can. Geol. Surv., Paper, 76-16:1-9.

GRASTY, R.L. \& DARNLEY, A.G. 1971. The calibration of gamma-ray spectrometer for ground and airborne use. Can. Geol. Surv., Paper, spectrom.

KILLEEN, P.G. 1979. Gamma-ray spectrometric methods in uranium A817

exploration - application and interpretation. In: HOOD, P.J. ed. Recebido em 22 de novembro de 1993

Geophysics and Geochemistry in the SearchforMetallic Ores. Geol. Revisão do autor em 14 de março de 1994 Surv. Can. (Economic Report 255). Revisão aceita em 28 de março de 1994
MINTY, B.R.S. 1988. A Review of Airborne Gamma-Ray Spectrometry Resources. (Report 255).

\section{MANUSCRITO}

